\title{
Modularities Maximization in Multiplex Network Analysis Using Many-Objective Optimization
}

\author{
Asep Maulana \\ Leiden Institute of Advance Computer Science LIACS, \\ Leiden University \\ Niels Bohrweg 1-2333 CA Leiden, The Netherlands \\ a.asep.maulana@liacs.leidenuniv.nl \\ Valerio Gametto \\ Lorentz Institute for theoretical physics, \\ Leiden University \\ Niels Bohrweg 2-2333 CA Leiden, The Netherlands
}

\author{
Diego Garlaschelli \\ Lorentz Institute for theoretical physics, Leiden \\ University, \\ Niels Bohrweg 2-2333 CA Leiden, The Netherlands
}

Iryna Yevesyeva

Faculty of Technology, Gateway House 5.33,

De Montfort University

Leicester, LE1 9BH, United Kingdom

\author{
Michael Emmerich \\ Leiden Institute of Advance Computer Science LIACS, \\ Leiden University \\ Niels Bohrweg 1-2333 CA Leiden, The Netherlands
}

\begin{abstract}
Nowadays, social network analysis receives big attention from academia, industries and governments. Some practical applications such as community detection and centrality in economic networks have become main issues in this research area. Community detection algorithm for complex network analysis is mainly accomplished by the Louvain Method that seeks to find communities by heuristically finding a partitioning with maximal modularity. Traditionally, community detection applied for a network that has homogeneous semantics, for instance indicating friend relationship between people or import-export relationships between countries etc. However we increasingly deal with more complex network and also with so-called multiplex networks. In a multiplex network the set of nodes stays the same, while there are multiple sets of edges. In the analysis we would like to identify communities, but different edge sets give rise to different modularity optimizing partitions into communities. We propose to view community detection of such multilayer networks as a many-objective optimization problem. For this apply Evolutionary Many Objective Optimization and compute the Pareto fronts between different modularity layers. Then we group the objective functions into community in order to better understand the relationship and dependence between different layers (conflict, indifference, complementarily). As a case study, we compute the Pareto fronts for model problems and for economic data sets in order to show how to find the network modularity tradeoffs between different layers.
\end{abstract}

Keywords-Multiplex networks, community detection, manyobjective optimization, modularity maximization

\section{INTRODUCTION}

In many disciplines complex systems can be studied through network modeling and analysis. This yields a better understanding of complex phenomena, including conflicting sociology phenomena, spreading of disease, conflicting economic situations, telecommunication systems, biological systems, and networks in engineering. The networks or a collection of nodes are joined in pairs by edges. Clustering such groups of nodes in the network has become an important part of research. Network data becomes increasingly available but also complex due to the omnipresence of data measurement and inquiry as a recent trend.

In this work we will focus on a special class of networks so-called multiplex networks. Often for the same set of network nodes, several or many network layers can be defined. Network defining trade in different types of commodities is an example and it will provide a case study for this paper. Other examples include multiplex networks

- in communication via different channels (social media, telephone, peer-to-peer),

- in biology, the different types of signaling networks of trees or plants (via scents, via insects, via underground root networks),

- in sociology, defined by different types of relationships, such as personal friendship, relatives, business relationships, which might partially overlap.

This paper presents a first step into the analysis of such multiplex networks by means of modularity optimization, where modularity is a measure of the quality of how well a partition of a network is representing communities[MU1]. We consider the optimization of modularity for the different layer as the objective functions. Optimizing several $(2,3)$ objectives simultaneously can be addressed by multi-objective optimization and many $(>3)$ objectives by many-objective optimization resulting in a high dimensional Pareto front. By computing the Pareto fronts of pairs of different layers we find relationships between the objectives. Layers can be 'in conflict' with each other, meaning that they yield very different optimal 
modularity structures. They can be also complementary, meaning that maximizing the modularity of the one layer also maximizes the modularity of the other layer. In this case it is possible to merge the layers without losing essential information. Finally, it is also possible that the maximization of modularity of one layer does not affect the optimization of the modularity of another layer, in which case the problem could be easily decomposed.

\section{RELATED WORK}

To optimize many objectives simultaneously various approaches have been developed. Some of them aim at reducing complexity, such as Objective Reduction in Many-objective Optimization: Linear and Nonlinear Algorithms [3], Reducing Complexity in Many Objective Optimization Using Community Detection [4], and Objective Reduction Based on Nonlinear Correlation Information Entropy [5]. Other approaches are based on Evolutionary Multiobjective optimization (EMO) extended to deal with many objectives, cf. [2].

In this paper we will follow the approach by Maulana et al. [4]. They proposed a method to reduce complexity in many objective optimization by forming groups of objective functions based on the correlation between objective functions: Objectives are either conflicting (negative correlation), supporting each other (positive correlation), or indifferent w.r.t. each other (zero correlation) - here assuming that all objectives are to be maximized (or all objectives are to be minimized). Based on the correlation matrix one can form groups of networks with similar structure and oppose them to groups with conflicting structure. Moreover independent sub-problems can be separated. Thereby one can reduce the complexity of the overall multiple modularity maximization problem and gain insight into the structure of the many-objective problem.

\section{PRELIMINARIES}

Next we will introduce a formal definition of a multiplex network, modularity optimization and many objective optimization.

For comparative study, we apply NSGA-II [16] and SMSEMOA [MU2]|[14,15 ][MU3]algorithms.
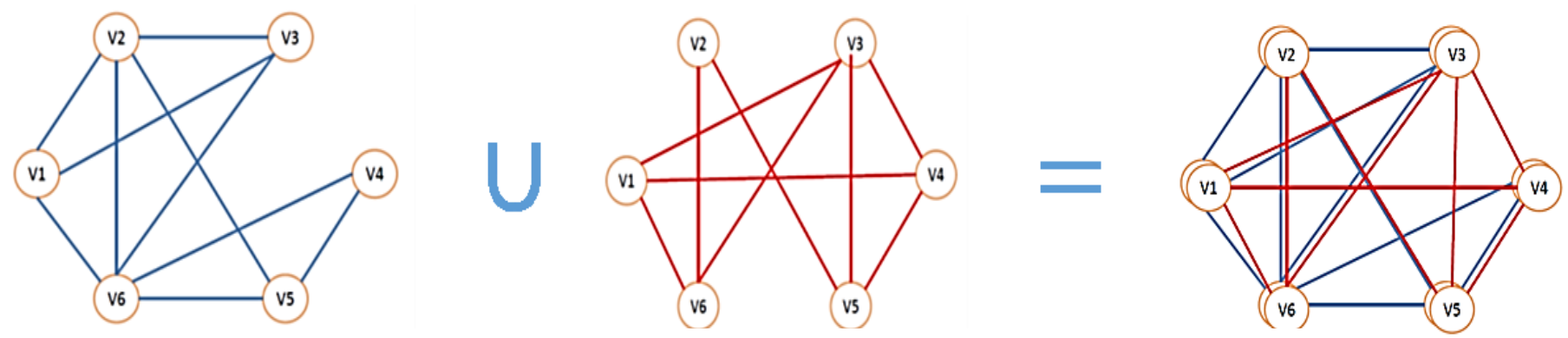

Figure 1 A visual illustration of multiplex network consists of two layers of networks distinguished by blue and red colour. Each layer has different type of links but nodes remain the same. The union of these layers, indicated by $U$, forms the multiplex network. 
$m$ denote the number of nodes, and $k_{i}=\sum_{j} A_{i j}$ denote the sum of weights of edges belonging to node $i$. Moreover, $c_{i}$ is the community to which node $i$ is assigned. Finally $\delta(.,$.$) is the$ Kronecker symbol, which is equal to 1 if and only if both arguments are equal to each other. Otherwise it obtains the value of 0 . Now the modularity is defined formally as:

$$
Q=\frac{1}{2 m} \sum_{i j}\left[A_{i j}-\frac{k_{i} k_{j}}{2 m}\right] \delta\left(c_{i}, c_{j}\right)
$$

It can be shown by polynomial reduction of 3PARTITION that modularity maximization is NP complete [6], and thus, in general, is difficult to solve this problem exactly. There are however several fast heuristics available, such as the Louvain method [1], which is a greedy heuristic that finds high modularity partitions of a network in short time. The first phase of Louvain method begins by placing each node in its own singleton 'community'. Then the looping over all nodes is done in the following way:

For each node $i$ all neighbors, that is, all the nodes $j$ such that $A_{i j}$ is nonzero, are analyzed from the point of view of the gain computed after removing $i$ from its community and placing it in the community of $j$. The node $i$ is then put in the community for which the increase in modularity is largest. If none of the potential re-assignments of $i$ into other communities is associated with positive gain in modularity, $i$ stays in its original community and the algorithm moves on to the next node. The loop is repeated until no further improvements are obtained, i.e. when the modularity has reached a local optimum. In the next phase of the algorithm, a new network is constructed with the communities of nodes obtained at the first phase of the Louvain method. The weights of the edges between the new nodes are given by the sum of the weights between all nodes between communities of the previous phase. When this phase is finished, a new phase is started, and so on. This creates a hierarchy of communities. The algorithm stops when a maximum of the modularity is obtained, or in practice, when the last performed pass did not further increase modularity.

\section{Many-Objective Optimization}

A multi-objective optimization problem is defined by a number of objective functions $f_{i}: X \rightarrow \mathbb{R}, i=1, \ldots, m$ to be maximized (or maximized) for some search space $X$. A solution $a \in X$ is said to dominate a solution $b \in X$ if and only if $\forall i: f_{i}(a) \geq f_{i}(b)$ and $\exists j: f_{j}(a)>f_{j}(b)$. Two solutions in $a \in X$ and $b \in X$ are non-dominated w.r.t. each other if neither $a$ dominates $b$ nor $b$ dominates $a$. The efficient set $X_{e}$ is the set of solutions in $X$ that is not dominated by any solution in $X$. The Pareto front $P F$ is the image set of $X_{e}$, i.e. $P F=\{f(x) \mid x \in X\}$.

Many-objective optimization (MOP) applies to problems with more than three objective functions [7]. The research problem of optimizing modularities in multiplex networks discussed in this work is formulated as a MOP. The two main issues differentiating many-objective problems from multi-objective problems are the following: on the one hand, a large number of objective functions makes visualization of the Pareto front impractical; on the other hand, analysis of Pareto fronts is difficult due to the tendency that a majority of solutions will be non-dominated. Hence, the tradeoff analysis of conflicts between objective functions and the representation of the entire Pareto front can become difficult and in-transparent. Moreover, a high number of objectives can yield to a significant increase in the computational time complexity required to compute Pareto fronts. In spite of these difficulties many technique and approach have been tried to deal with many objective optimization $[2,3,4,5]$.

\section{Problem DefFinitation of Many-ObJective COMMUNITY DETECTION IN MULTIPLEX NETWORK}

Our research approach is to perform many-objective optimization of network modularity by computing and visualizing a matrix of Pareto fronts for pairs of objectives. Then we use community detection algorithms to group objective functions in order to understand and visualize the conflict or correspondence of community structures w.r.t. different edge sets. For every edge set one objective function is defined, which is to maximize the modularity for this edge set. The search space $X$ is the space of all partitionings of the node sets. In this way for a multiplex network $G$ with layers $G_{1}, \ldots, G_{M}$ we define $M$ objective functions $Q_{1}: X \rightarrow$ $\mathbb{R}_{0}^{+}, Q_{2}: X \rightarrow \mathbb{R}_{0}^{+}, \ldots, Q_{M}: X \rightarrow \mathbb{R}_{0}^{+}$. All objective functions are to be maximized.

Our first goal is to compute Pareto optimal solutions. Then we analyze projections to pairs of objective functions (corresponding to pairs of layers), in order to understand the relationship between layers in terms of modularity structure. In this way we aim to gain insight into essential aspects of the community structure of a given multiplex network.

\section{NETWORK ANALYSIS METHOD}

Given as an input a multiplex network with $M$ layers represented by a set of graphs $G_{1}, \ldots, G_{M}$, the approach is called Pareto front Modularity for Multiplex Network (PaMoPlex ). It is summarized in a work flow which consists of two major phases: (1) Preparation of data by optimization, (2) Analysis of data.

The preparation of data in step (1) of the analysis consists of solving optimization tasks to find non-dominated solutions. In order to get more precise results we also compute single objective optima and marginal Pareto fronts for every pair of two objective functions (between the modularities (objectives) 
associated with two layers, each). [MU5]The first phase is summarized in the next three steps:

- Single Objective Optimization: Optimize the modularity of each network separately using evolutionary single objective optimization based on a genetic algorithm.

- Many Objective Optimization: Optimize the modularity of network, all layers together, as one unity in multiplex network. For this we use a $M$-objective many optimization algorithm[MU6].

- Pairwise Pareto-Front Computation: Optimize modularity for pairs of objectives.

The optimization method is evolutionary multi-objective optimization based on NSGA-II [16] and SMS EMOA [14,15]. (population size: 100, number of generations: 2000). For small examples, we use complete enumeration of partitions.

In the second phase the obtained data are analyzed. This is conducted in the following three steps:

- Matrix of Pareto Fronts Analysis: Visualization of Pareto Fronts is done on a plot matrix, where each tile with $j \in\{1, \ldots, M\}, i \in\{1, \ldots, M\}, j>i$ consists of a plot of a Pareto front of tradeoffs between objectives $Q_{i}$ and $Q_{j}$. (see Figures 5).

- Correlation Heat map Analysis: Computation of the correlation coefficients matrix from the projections of the output of many-objective optimization. The heat map has as many rows and columns as the number of network layers (or objectives). The Pearson correlation coefficients of the projected 2-objective function vectors have values in the range of $[-1,1]$ for each pair of objective functions; see Table 2 for an example. In the heat map, see Figure 6 for an example, blue color represents negative correlations, whereas red color represents positive correlations. The intensity (darkness) and size of the colored square in each matrix cell grows with the absolute value.

- Community Analysis: This tool is based on the result of the correlation analysis. The correlation matrix is used for community detection by the graph theoretic algorithm to detect communities using the information of correlation coefficients matrix and interpreting it as edge weights. Here the analysis proposed by Maulana et al. [4] is used, where the edge weight is determined by the absolute value of the correlation coefficient. This leads to a separation of independent communities of layers. Conflicting communities are placed opposite to each other (see Figure 7).

Further details on the analysis on examples and interpretation of results will be discussed in the subsequent sections.

\section{EXAMPLE}

\section{A. Analysis on Synthesized Multiplex Networks}

As an illustrative example on how to interpret results of multiobjective modularity optimization, we computed the exact Pareto fronts for three synthesized multiplex networks consisting of only two layers each. The networks and the corresponding Pareto fronts are displayed in Figure 2, 3 and 4. Red edges denote edge weights of 3 , blue edges represent edge weights 1 , and omitted edges have weight 0 . Complete enumeration of all 203 possible portioning was used to compute the exact Pareto fronts (cf. Bell 1934 [8]).

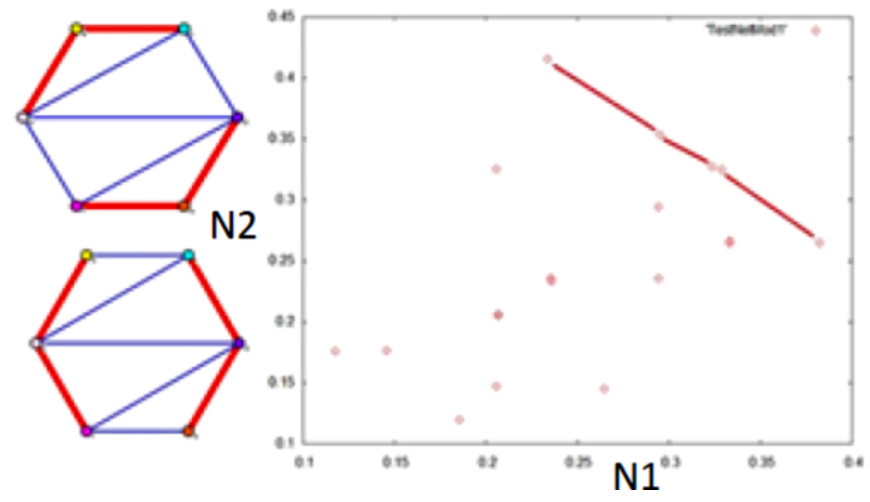

Figure $2 \mathrm{~A}$ visual depiction of Pareto front for network modularity between two network layers N1 and N2 corresponding to highly conflicting objectives $\mathrm{O}_{1}$ and $\mathrm{O}_{2}$

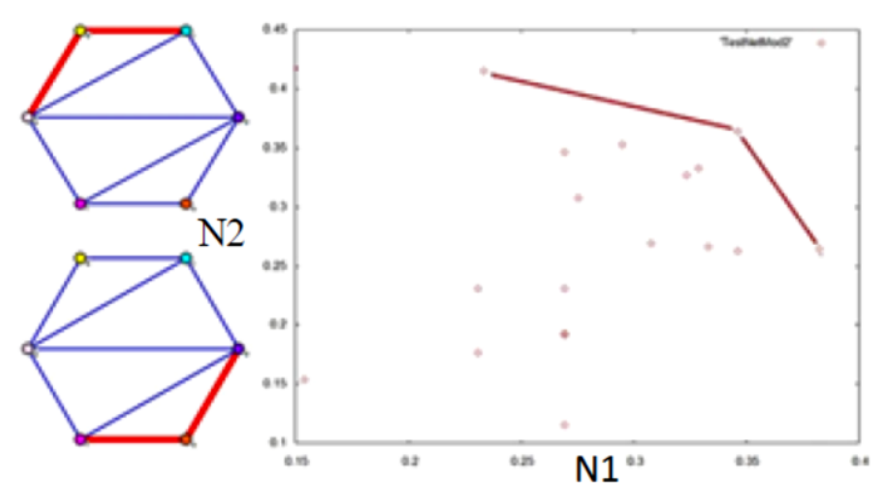

Figure $3 \mathrm{~A}$ visual depiction of Pareto front for network modularity between two networks N1 and N2 corresponding to highly correlated objectives $\mathrm{O}_{1}$ and $\mathrm{O}_{2}$

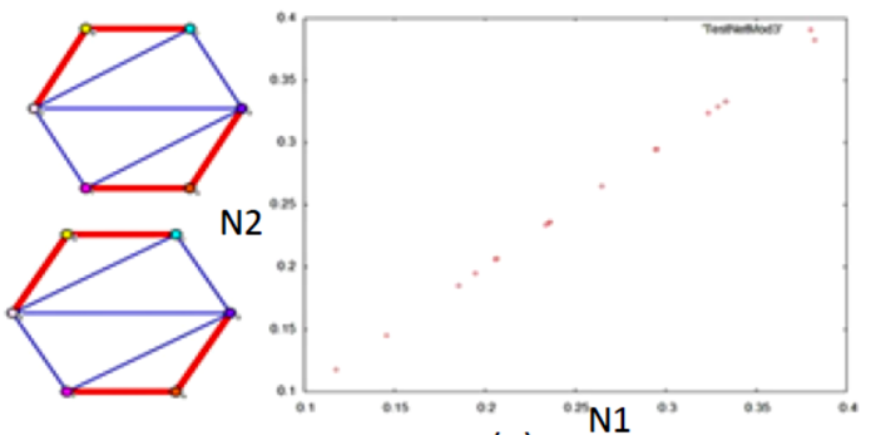


Figure 4 A visual depiction of Pareto front for network modularity between two networks $\mathrm{N} 1$ and $\mathrm{N} 2$ corresponding to objectives $\mathrm{O}_{1}$ and $\mathrm{O}_{2}$ with exact modularity

The first network in Figure 2 is a multiplex network where the maximization of modularity is conflicting, due to nonoverlapping communities w.r.t. both layers. The linear Pareto front indicates a strong conflict between the maximization of two types and it is difficult to find a compromise solution that optimizes both objectives at the same time.

In the second example, in Figure 3, the optimal modularity for the first network is achieved by grouping the upper nodes in the graph, while for the second network it is important to group the lower nodes. Thereby the value of the modularity is widely indifferent to how the remaining nodes are grouped. This represents a case where the modularity optimization for the two layers is almost independent and the Pareto front has a knee point solution where both objective functions almost obtain their maximum. The correlation is close to zero.

Finally, the third example in Figure 4 shows a multiplex network consisting of two equal edge sets. Here solutions can be found that cluster for one layer optimally w.r.t. modularity necessarily also do so for the modularity of the second network. In other words, optimizing one network coincides with optimizing the other network. This is indicated by a perfect correlation between the modularities of sampled points - even for random inputs. The Pareto front consists of only a single solution.

In real world applications, it is of course not so obvious how the structure of the Pareto front looks like. These three examples should be seen as boundary cases, which can help to interpret and understand the observed shape of Pareto fronts in such real world networks.

\section{B. Economic Trade Multiplex Network Analysis}

Next, a full PaMoPlex analysis on a economic dataset is provided. The data originates from network economy (trade data) using import-export commodities network between countries in 2011. (see [11], Appendix) The data represents the import-export relationships between some countries of the world, disaggregated for different traded commodities. This network can be defined as a multiplex network composed by many layers, where each layer is given by a different commodity. The nodes are given by 207 countries. A link between two countries in a $i$-th layer defined as weight will exists if there is trade between them in the $i$-th commodity, for $i \in\{1, \ldots, 11\}$. Data are presented in matrix form: rows and columns represent countries, and the entries of the matrices are the volumes of trade. It is therefore a weighted multiplex network. The general classification is based on 96 [MU7][e8]different commodities. The classification performed by grouping together similar commodities; this procedure leads to 11 aggregated 'super-commodities'.

\begin{tabular}{|c|c|c|c|c|}
\hline & \multicolumn{2}{|c|}{$\begin{array}{c}\text { Modularity based on } \\
\text { standard Louvain } \\
\text { method } \\
\text { network }\end{array}$} & \multicolumn{2}{|c|}{$\begin{array}{c}\text { Single objective } \\
\text { optimization } \\
\text { by Genetic } \\
\text { Algorithm }\end{array}$} \\
\cline { 2 - 5 } & Modularity & $\begin{array}{c}\text { Number } \\
\text { of } \\
\text { cluster }\end{array}$ & $\begin{array}{c}\text { Modulari } \\
\text { ty }\end{array}$ & $\begin{array}{c}\text { Number } \\
\text { of } \\
\text { cluster }\end{array}$ \\
\hline Trade 1 & 0.34392 & 9 & 0.35162 & 9 \\
\hline Trade 2 & 0.34794 & 9 & 0.35225 & 9 \\
\hline Trade 3 & 0.30513 & 9 & 0.30801 & 8 \\
\hline Trade 4 & 0.33691 & 7 & 0.33771 & 7 \\
\hline Trade 5 & 0.29084 & 6 & 0.29968 & 6 \\
\hline Trade 6 & 0.26811 & 5 & 0.27008 & 5 \\
\hline Trade 7 & 0.24781 & 7 & 0.24873 & 7 \\
\hline Trade 8 & 0.18622 & 6 & 0.18863 & 5 \\
\hline Trade 9 & 0.29881 & 5 & 0.29882 & 4 \\
\hline Trade 10 & 0.22961 & 5 & 0.22966 & 4 \\
\hline Trade 11 & 0.15493 & 4 & 0.15494 & 4 \\
\hline
\end{tabular}

Table 1 A modularity for each single network and optimization result for those modularity based on single objective optimization using genetic algorithm

The single objective optimization was conducted by a genetic algorithm and by the Louvain method. In all cases the genetic algorithm found a better result. The results are summarized in Table 1. A typical number of communities when maximizing modularity are between 5 and 9 .

The genetic algorithm is from the software package JMetal (gGA). It has population size 2000 and 100 generations were conducted. The default parameter settings for the genetic operators were used (http://jmetal.sourceforge.net/, February 2015). We suppose that by tuning of parameters better results can be achieved, but defer such studies to future research in order to focus more on the overall analysis method in this paper. The many-objective optimization yields a Pareto front that is embedded in an 11 dimensional space. The analysis of the correlation and community between objectives was conducted after the approach mentioned in [4].

From this we compute the heat map of correlation between objectives (Figure 6) and the community structure (Figure 7). The results are also reflected in the Pareto front plot matrix (Figure 5). Our interpretation of results is as follows: Strong conflicts occur between $Q_{3}$ and $Q_{8}, Q_{3}$ and $Q_{9}, Q_{1}$ and $Q_{8}, Q_{4}$ and $Q_{5} \cdot Q_{1}$ and $Q_{2}, Q_{1}$ and $Q_{3}, Q_{4}$ and $Q_{11}, Q_{4}$ and $Q_{10}, Q_{4}$ and $Q_{11}$. 


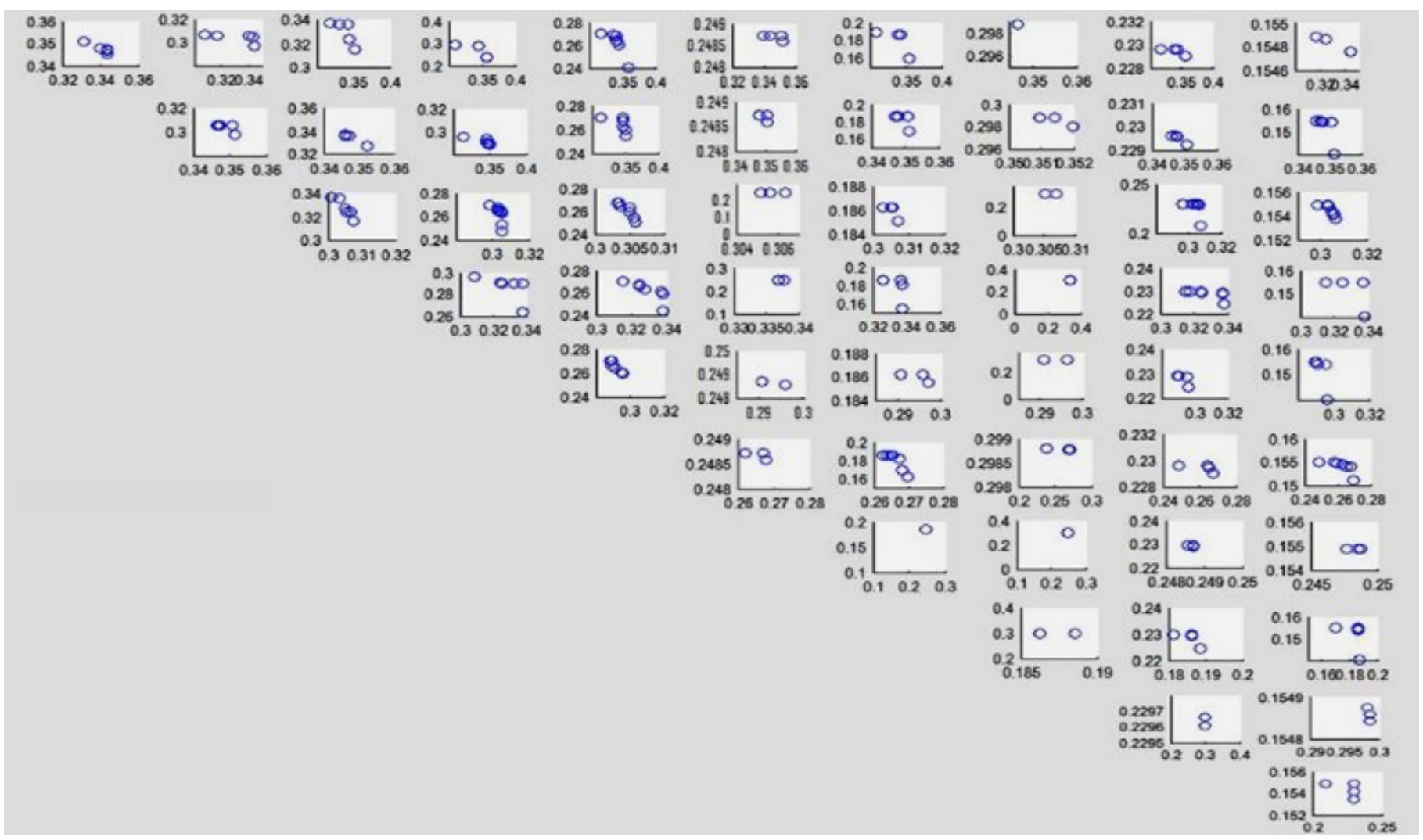

Figure 5 Pairwise Pareto Fronts Matrix for Economic Trade Network Analysis

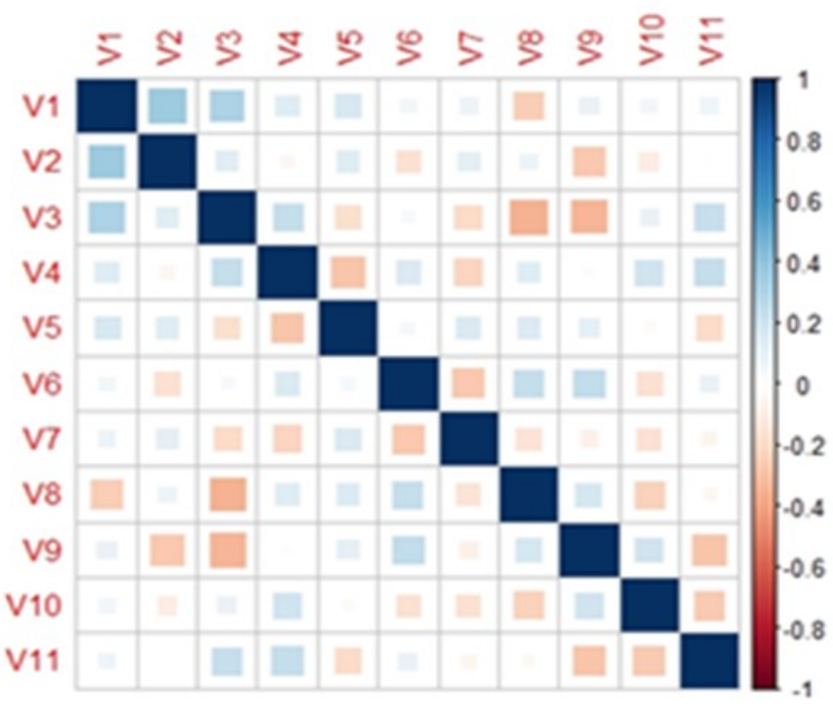

Figure 6 Correlation heat map for many-objective optimizations of 11 node trade network

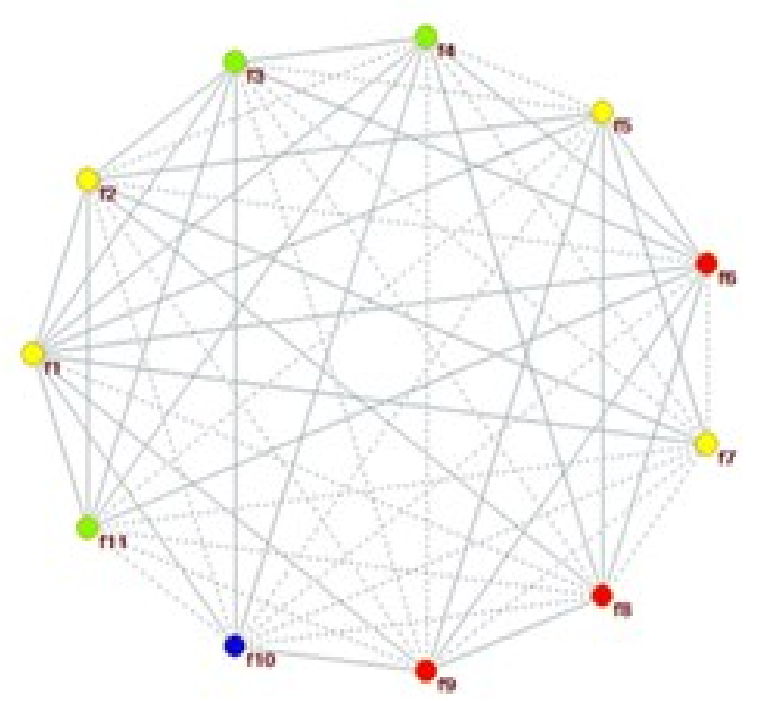

Figure 7 community structure for many-objective optimizations of 11 node trade network 
From the analysis we can for instance conclude that for tradenetworks of $Q_{3}$ and $Q_{8}$ the countries cannot be clustered in a way that community structures for both groups of commodities are well represented. On the contrary, for $Q_{1}$ and $Q_{2}$ there exists a clustering that represents the community structures for both communities very well. For the sake of completeness we note that $Q_{1}$. (see Appendix for commodity description). It seems logical that the mainly agricultural products of group $Q_{1}$ and $Q_{2}$ appear to adhere to similar trade community structures, whereas for the very disjoint products in group $Q_{3}$ and $Q_{8}$, it might have been difficult to predict a priori how their trade networks will overlap.

\section{CONCLUSION AND FUTURE WORK}

The paper showed how to apply many-objective optimization for the analysis of multiplex networks. Different ways on how to analyze the community structure in multilayer networks were shown, all relying upon data from many-objective optimization. First we discuss the meaning of the Pareto fronts between modularities by exact computations of Pareto fronts on three illustrative examples, which represent important boundary cases. Then, on the example of trade networks for commodities, we perform a full analysis. First we generate data using manyobjective optimization, biobjective optimization (of any pair of layers), and single objective optimization (of any single layer). The results are analyzed using three tools suggested here:

Correlation heatmap, community of objectives analysis, and Pareto-front plot matrix. These were computed for an economic trade network with 11 groups of commodities. Clearly a grouping emerges in terms of complementarity and/or in terms of indifference. NSGA-II, SMS-EMOA and single-objectve genetic algorithms can be used as a search engine. In the future a more in-depth analysis on an extended benchmark will be conducted. Moreover, it is promising to also look at other network metrics, such as centrality, to gain further insights into multiplex network structure.

\section{Appendix}

Description on selected commodities in trade network:

We will not go in detail about all commodities, due to space limitations, but briefly describe those mentioned above:

$Q_{1}=$ LLive animals, Meat and edible meat offal, Fish, crustaceans and acquatic invertebrates, Dairy produce; birs eggs; honey and other edible animal products

$Q_{2}=$ LLive trees, plants; bulbs, roots; cut flowers and ornamental foliage tea and spices; Edible vegetables and certain roots and tubers; Edible fruit and nuts; Citrus fruit or melon peel; Coffee, tea, mate and spices; Cereals; Milling products; malt; starch; inulin; wheat gluten; Oil seeds and oleaginous fruits; miscellaneous grains, seeds and fruit; Industrial or medicinal plants; straw and fodder

$Q_{3}=\{$ Lac; gums, resins and other vegetable sap and extracts Vegetable plaiting materials and other vegetable products; Animal, vegetable fats and oils, cleavage products, etc; Edible preparations of meat, fish, crustaceans, mollusks or other aquatic invertebrates; sugars and sugar confectionary; Cocoa and cocoa preparations; Preparations of cereals, flour, starch or milk; bakers wares; Preparations of vegetables, fruit, nuts or other plant parts; Miscellaneous edible preparations; Beverages, spirits and vinegar; Food industry residues and waste; prepared animal feed; Tobacco and manufactured tobacco substitutes

$Q_{8}=\{$ Carpets and other textile floor coverings; Special woven fabrics; tufted textile fabrics; lace; tapestries; trimmings; embroidery; Impregnated, coated, covered or laminated textile fabrics; textile articles for industrial use; Knitted or crocheted fabrics; Apparel articles and accessories, knitted or crocheted; Apparel articles and accessories, not knitted or crocheted; Other textile articles; needlecraft sets; worn clothing and worn textile articles; rags; Footwear, gaiters and the like and parts thereof; Headgear and parts thereof; Umbrellas, walking sticks, seat sticks, riding crops, whips, and parts thereof; Prepared feathers, down and articles thereof; artificial flowers; articles of human hair

(See COMTRADE 96 Classification of commodities for 2011 on http://comtrade.un.org/db/mr/rfCommoditiesList.aspx)

Moreover we use the following grouping of commodities

- from 1 to 5: comm 01;

- from 6 to 12: comm 02

- from 13 to 24 : comm 03

- from 25 to 35 : comm 04

- from 36 to 40: comm 05

- from 41 to 49 : comm 06

- from 50 to 56 : comm 07

- from 57 to 67 : comm 08

- from 68 to 82 : comm 09

- from 83 to 88 : comm 10

- from 89 to 96 : comm 11

The commodity data we used was from 2011 for all 207 countries.

\section{Acknowledgements:}

Asep Maulana greatfully acknowledges financial support by the Indonesian Endowment Fund for Education (LPDP). 


\section{REFERENCES}

[1] Newman, M. E. (2006). Modularity and community structure in networks. Proceedings of the national academy of sciences, 103(23), 8577-8582.

[2] Knowles, J., \& Corne, D. (2007). Quantifying the effects of objective space dimension in evolutionary multiobjective optimization. In Evolutionary multi-criterion optimization (pp. 757-771). Springer Berlin/Heidelberg.Chicago.

[3] Saxena, D. K., Duro, J. A., Tiwari, A., Deb, K., \& Zhang, Q. (2013). Objective reduction in many-objective optimization: Linear and nonlinear algorithms. Evolutionary Computation, IEEE Transactions on, 17(1), 77-99.

[4] Maulana, A., Jiang, Z., Liu, J., Bäck, T., \& Emmerich, M. (2015, May). Reducing complexity in many objective optimization using community detection. In Evolutionary Computation (CEC), 2015 IEEE Congress on (pp. 3140-3147). IEEE.

[5] Wang, H., \& Yao, X. (2015). Objective reduction based on nonlinear correlation information entropy. Soft Computing, 115.

[6] Brandes, U., Delling, D., Gaertler, M., Görke, R., Hoefer, M., Nikoloski, Z., \& Wagner, D. (2006). On modularity-npcompleteness and beyond. Univ., Fak. für Informatik, Bibliothek.

[7] Fleming, P. J., Purshouse, R. C., \& Lygoe, R. J. (2005, March). Many-objective optimization: An engineering design perspective. In Evolutionary multi-criterion optimization (pp. 14-32). Springer Berlin Heidelberg.

[8] Bell, E. T.: Exponential Numbers, Amer. Math. Monthly 41(1934), no. 7, 411-419. MR 1523147, http://dx.doi.org/10.2307/2300300 - See more at: http://www.ams.org/journals/mcom/1962-16-080/S0025-57181962-0148604-2/\#sthash.EeulVfte.dpuf

[9] M. Kivelä, A. Arenas, M. Barthelemy, J. P. Gleeson, Y. Moreno, and M. A. Porter, Journal of Complex Networks 2, 203 (2014)

\begin{tabular}{|c|c|c|c|c|c|c|c|c|c|c|}
\hline 1 & 0.3548 & 0.3059 & 0.1378 & 0.1695 & 0.0577 & 0.0759 & -0.2474 & 0.0891 & 0.0578 & 0.0695 \\
\hline 0.3548 & 1 & 0.1247 & -0.0447 & 0.1351 & -0.1596 & 0.1172 & 0.0715 & -0.2689 & -0.0937 & 0.0017 \\
\hline 0.3059 & 0.1247 & 1 & 0.2332 & -0.17 & 0.0345 & -0.1839 & -0.3465 & -0.3371 & 0.0853 & 0.2283 \\
\hline 0.1378 & -0.0447 & 0.2332 & 1 & -0.279 & 0.1558 & -0.2159 & 0.1382 & -0.0122 & 0.1928 & 0.2393 \\
\hline 0.1695 & 0.1351 & -0.17 & -0.279 & 1 & 0.0468 & 0.1544 & 0.1428 & 0.1049 & -0.0278 & -0.1891 \\
\hline 0.0577 & -0.1596 & 0.0345 & 0.1558 & 0.0468 & 1 & -0.2646 & 0.2314 & 0.2439 & -0.1592 & 0.081 \\
\hline 0.0759 & 0.1172 & -0.1839 & -0.2159 & 0.1544 & -0.2646 & 1 & -0.1495 & -0.0774 & -0.1515 & -0.0504 \\
\hline-0.2474 & 0.0715 & -0.3465 & 0.1382 & 0.1428 & 0.2314 & -0.1495 & 1 & 0.1715 & -0.2256 & -0.0349 \\
\hline 0.0891 & -0.2689 & -0.3371 & -0.0122 & 0.1049 & 0.2439 & -0.0774 & 0.1715 & 1 & 0.1913 & -0.2738 \\
\hline 0.0578 & -0.0937 & 0.0853 & 0.1928 & -0.0278 & -0.1592 & -0.1515 & -0.2256 & 0.1913 & 1 & -0.2531 \\
\hline 0.0695 & 0.0017 & 0.2283 & 0.2393 & -0.1891 & 0.081 & -0.0504 & -0.0349 & -0.2738 & -0.2531 & 1 \\
\hline
\end{tabular}

Table 2 A correlation Matrix of modularity for 11 node trade network based on many-objective optimization by SMS-EMOA algorithm.
[10] S. Boccaletti, G. Bianconi, R. Criado, C. I. del Genio, J. Gomez-Gardeñes, M. Romance, I. Sendiña Nadal, Z. Wang, and M. Zanin. The structure and dynamics of multilayer networks. Physics Reports 544, 1 (2014).

[11] M. Barigozzi, G. Fagiolo, and D. Garlaschelli. Multinetwork of international trade: A commodity-specific analysis . Physical Review E 81 (2010).

[12] Didier, G., Brun, C., \& Baudot, A. (2015). Identifying communities from multiplex biological networks. PeerJ, 3, e1525.

[13] Hu, H., van Gennip, Y., Hunter, B., Bertozzi, A. L., \& Porter, M. A.(2012, December). Multislice modularity optimization in community detection and image segmentation. In 2012 IEEE 12th International Conference on Data Mining Workshops (pp. 934-936). IEEE.

[14] Wagner, Tobias, Nicola Beume, and Boris Naujoks. "Pareto-, aggregation-, and indicator-based methods in many-objective optimization." International conference on evolutionary multicriterion optimization. Springer Berlin Heidelberg, 2007.

[15] Beume, Nicola, Boris Naujoks, and Michael Emmerich. "SMS-EMOA: Multiobjective selection based on dominated hypervolume." European Journal of Operational Research 181.3 (2007): 1653-1669.

[16] Deb, Kalyanmoy, et al. "A fast and elitist multiobjective genetic algorithm: NSGA-II." IEEE transactions on evolutionary computation 6.2 (2002): 182-197. 
